\title{
O017. Cortical functional correlates of responsiveness to short-lasting preventive intervention with ketogenic diet (KD) in migraine: a multimodal evoked potentials study
}

\author{
Martina Bracaglia ${ }^{1 *}$, Gianluca Coppola², Cherubino Di Lorenzo ${ }^{1}$, Davide Di Lenola ${ }^{1}$, Francesco Pierelli ${ }^{1}$
}

From Abstracts from the 1st Joint ANIRCEF-SISC Congress

Rome, Italy. 29-31 October 2015

\section{Background}

Ketogenic diet (KD) - a dietetic regimen that mimics fasting in producing ketone bodies - seems to have a role in preventing migraine. The molecular mechanisms underpinning ketogenic diet effectiveness are only partially clear.

\begin{abstract}
Aim
With the aim of identifying cortical electrofunctional correlates of responsiveness to short-lasting preventive intervention with $\mathrm{KD}$ in migraine, we recorded visual (VEPs) and somatosensory (SSEPs) evoked-potentials before and after 1-month intervention with KD.
\end{abstract}

\section{Methods}

Sixteen interictal migraine without aura patients $(\mathrm{MO}$, ICHD-II code 1.1) underwent VEPs (right eye stimulation, 600 sweeps, $3.1 \mathrm{~Hz}$ reversal rate, $15 \mathrm{~min}$ of arc check) and median nerve SSEPs (right stimulation, 500 sweeps, 4.4 repetition rate, 1.2 motor threshold) recordings, before and during ketogenesis, confirmed by urinary sticks. We measured VEPs N75-P100 and SSEPs N20-P25 amplitudes respectively in 6 and in 2 sequential blocks of 100 sweeps, and habituation as the slope of the linear regression between block 1 to 2 for SSEPs or between 1 to 6 for VEPs.

\section{Results}

After 1-month of KD, a significant reduction of mean migraine frequency (from a mean of 4.1 to 1.4 attacks/

\footnotetext{
* Correspondence: martinabracaglia@alice.it

'Sapienza University of Rome Polo Pontino, Department of Medico-surgical

Sciences and Biotechnologies, Latina, Italy

Full list of author information is available at the end of the article
}

month, $\mathrm{p}<0.001$ ) and duration (from 51.9 to 16.3 hours/month, $\mathrm{p}<0.001$ ) was observed. KD did not change $1^{\text {st }}$ SSEP and VEP block of 100 sweeps, but significantly induced normalization of the interictally reduced VEPs (from +0.09 to $-0.14, \mathrm{p}=0.017$ ) and SSEPs (from 0.38 to $-0.48, \mathrm{p}=0.002$ ) habituation during the subsequent blocks.

\section{Conclusions}

We found evidence for KD-induced changes at cortical level in parallel to an improvement of migraine. Since KD was able to restore normal EPs habituation curves during stimulus repetition without changing significantly early amplitude responses, we hypothesize that KD acts on habituation via an enhancement of late GABA inhibition.

Written informed consent to publication was obtained from the patient(s).

\section{Authors' details}

${ }^{1}$ Sapienza University of Rome Polo Pontino, Department of Medico-surgical Sciences and Biotechnologies, Latina, Italy. ${ }^{2} \mathrm{G}$. B. Bietti Foundation-IRCCS,

Department of Neurophysiology of Vision and Neuro-ophthalmology, Rome, Italy.

Published: 28 September 2015

\section{doi:10.1186/1129-2377-16-S1-A58}

Cite this article as: Bracaglia et al:: O017. Cortical functional correlates of responsiveness to short-lasting preventive intervention with ketogenic diet (KD) in migraine: a multimodal evoked potentials study. The Journal of Headache and Pain 2015 16(Suppl 1):A58. 